\title{
Sensitivity of the Radial Velocity Technique in Detecting Outer Planets
}

\author{
J. A. Eisner \& S. R. Kulkarni \\ Palomar Observatory 105-24, California Institute of Technology, Pasadena, CA 91125
}

Received __; accepted _ 


\begin{abstract}
The technique of radial velocity (RV) has produced spectacular discoveries of short-period Jovian mass objects around a fraction (5 to 10\%) of nearby G stars. Although we expect Jovian planets to be located in long-period orbits of decades or longer (if our solar system is any guide), detecting such planets with RV technique is difficult due to smaller velocity amplitudes and the limited temporal baseline (5-10 yr) of current searches relative to the expected orbital periods. In this paper, we develop an analytical understanding of the sensitivity of RV technique in the regime where the the orbital period is larger than the total baseline of the survey. Moreover, we focus on the importance of the orbital phase in this "long-period" regime, and develop a Least Squares detection technique based on the amplitude and phase of the fitted signal.

To illustrate the benefits of this amplitude-phase analysis, we compare it to existing techniques. Previous authors (e.g. Nelson \& Angel 1998) have explored the sensitivity of an amplitude-only analysis using Monte Carlo simulations. Others have supplemented this by using the slope of the linear component of the fitted sinusoid in addition (e.g. Walker et al. 1995; Cumming et al. 1999). In this paper, we illustrate the benefits of Least Squares over periodogram analysis, and demonstrate the superiority of an amplitude-phase technique over previous analyses.
\end{abstract}




\section{Introduction}

Radial velocity surveys of nearby stars have been employed in the search for extra-solar planets for nearly two decades. An important element of these surveys is the sensitivity; i.e. what is the minimum-mass planet that can be detected at a given orbital period? This issue boils down to a question about how well one can detect periodic signals in a finitely sampled data set.

There are two main regimes of analysis: the "short-period" regime, characterized by $\tau \ll T_{0}$ and the "long-period" regime with $\tau \gg T_{0}$. Here $\tau$ is the orbital period and $T_{0}$ is the duration of the survey. The planetary objects found to date through the RV technique are short period objects, with periods less than a year or so (e.g. Mayor \& Queloz 1995; Marcy \& Butler 1996; Fischer et al. 1998). The sensitivity of RV searches is very well understood in this short orbital period regime and compact analytical formulae exist (Lomb 1976; Scargle 1982; Horne \& Baliunas 1986; Nelson \& Angel 1998; Cumming, Marcy, \& Butler 1999).

According to current theoretical prejudice, however, one expects giant planets to be primarily formed in the colder regions of the proto-planetary nebula, and thus we expect such objects to possess periods in the range of many years to centuries (Boss 1995). Unfortunately, it is well known that the sensitivity of RV technique is considerably worse for planets with large orbital periods. Earlier analyses in the long-period regime have primarily concentrated on estimating the sensitivity through simulations (e.g. Nelson \& Angel 1998, Cumming et al. 1999).

This paper has two goals: to provide analytical insight into the sensitivity of the RV technique in the long-period regime, and to address the issues of detection and detection efficiency. We note that almost all of the previous work has concentrated on setting upper limits and not addressed the issue of detection. It is also worth noting that much of the previous work has been based on the periodogram method (e.g. Scargle 1982; Horne \& Baliunas 1986; Walker et al. 1995; Cumming et al. 1999). Following, Nelson \& Angel (1998), we argue at various points in the paper why a Least Squares approach is preferable to a periodogram approach. Essentially the Least Squares approach, in contrast to the traditional periodogram approach, offers the most general method 
and needs no modifications in the long-period regime or in the sparse-data regime.

The plan of the paper is as follows. We summarize the Least Squares approach and derive the basic equations in $\S$. In $\oint$ 通 we provide analytical estimates for obtaining a detection in the absence of any signal in the short and long-period regimes. In $\$$ t we carry out simulations and obtain estimates for minimum detectable signals in the presence of noise. We conclude in $\S$.

\section{Radial Velocity Technique: Basic Equations}

For simplicity, we will assume circular orbits throughout this discussion. A planet in a circular orbit undergoes acceleration, and because the linear momentum of the system must be conserved, the star undergoes a reflex acceleration. It is this acceleration that directly informs us of the presence of the companion. However, the observable is the velocity:

$$
v(t)=A \sin (2 \pi t / \tau+\phi)+\gamma
$$

where the amplitude, $A$ is given by

$$
A=\left(\frac{2 \pi G M}{\tau}\right)^{\frac{1}{3}} \frac{M_{p} \sin i}{M_{*}} .
$$

Here, $\gamma$ is the radial velocity of the planetary system, $\phi$ is the orbital phase, $\tau$ is the orbital period, $M_{p}$ is the planet mass, $M_{*}$ is the stellar mass, $M=M_{p}+M_{*} \approx M_{*}$ (as $M_{p} \ll M_{*}$ ), and $i$ is the inclination of the orbit with respect to the plane of the sky.

The sensitivity of an RV experiment is essentially defined as the minimum mass planetary companion that can be detected at a given period. For a given measurement of $A$, we invert Equation 2 to obtain a relation between $M_{p}$ and $\tau$ :

$$
M_{p} \sin i=A\left(\frac{M_{*}^{2}}{2 \pi G}\right)^{\frac{1}{3}} \tau^{\frac{1}{3}} .
$$

We see that $M_{p} \propto \tau^{\frac{1}{3}}$, which means that our sensitivity decreases as we look for longer-period orbits. Thus, it is not surprising that the first detection of an extra-solar planet was a planet with 
very short orbital period (Mayor \& Queloz 1995). There is an additional effect which makes it difficult to identify planets with long periods. With only a baseline of a few years, the RV surveys can only observe some fraction of $A$ for orbital periods longer than the observing baseline. In this (long-period) regime, the sensitivity depends critically on the orbital phase. The most sensitive mass estimates are obtained when the acceleration in the radial direction attains the extreme value. In contrast, when the radial acceleration is close to zero, the lack of curvature in the orbit makes the signal difficult to distinguish from the unknown systemic velocity of the system. These two effects combine to explain why all the planetary detections to date have orbital periods of less than 3 years (Mayor \& Queloz 1995; Marcy \& Butler 1996; Butler \& Marcy 1996; Butler et al. 1997; Noyes et al. 1997; Cochran et al. 1997; Marcy et al. 1998; Fischer et al. 1998; Mayor et al. 1998; Marcy et al. 1999; Queloz et al. 1999).

\subsection{Least Squares Fitting of Sinusoids}

The basic RV analysis consists of fitting the observations to the model specified in Equation 1 and then inferring the mass of the planet[s] from the fitted values through Equation 3. As noted by several authors (e.g. Scargle 1982; Horne \& Baliunas 1986; Nelson \& Angel 1998) the most optimal fitting method is obtained by using the technique of Least Squares. To enable the use of linear Least Squares fitting techniques we proceed by deriving a linear model equation from Equation 1:

$$
v(t)=v_{c} \cos \left(\omega t_{i}\right)+v_{s} \sin \left(\omega t_{i}\right)+\gamma
$$

here $v_{c}=A \sin \phi, v_{s}=A \cos \phi$ and $\omega=2 \pi / \tau$.

There appears to be considerable discussion and debate about the $\gamma$ term in the literature (e.g. Walker et al. 1995; Cumming et al. 1999). The origin of this debate can be traced to two issues. First, it is an assumption of periodogram analyses that the signal is a pure sinusoidal wave (i.e. $\gamma=0$ ). Indeed, the classic periodogram problem is the sine wave (with no offset) buried in zero-mean noise. The second issue is that astronomers are rarely interested in determining the 
precise radial velocity of a star and its planetary system-i.e. the systemic motion. For this reason, $\gamma$ is usually seen as a "nuisance" parameter. In some periodogram analyses, $\gamma$ is first determined from the mean of the data and in others $\gamma$ is determined in conjunction with $v_{c}$ and $v_{s}$. Cumming et al. (1999) refer to these as "fixed" and "floating" mean methods, respectively while Walker et al. (1995) call them "correlated" and "uncorrelated". However, our view is that such methods for including $\gamma$ (and the additional extension of including a constant acceleration term; see $\$ 4.3$ ) only make the periodogram closer to the Least Squares method. Thus, on philosophical grounds of generality we prefer the Least Squares approach. We justify this choice in greater detail below.

Essentially the basic fact is that $\gamma$ is needed to represent the physical model correctly. $\gamma$ may be an uninteresting parameter, but it is as unknown as $v_{c}, v_{s}$ and $\tau$. $\gamma$ can be dropped from Equation 1 only if it can be demonstrated that it is not covariant with the remaining three parameters, $v_{c}, v_{s}$ and $\tau$. As shown below, this is not the case and thus one must solve for all the parameters simultaneously. In astrometry, the problem is considerably worse with the position and proper motion being covariant with the parameters of a potential planetary orbit. As with the RV case, one must solve for all unknown parameters simultaneously rather than sequentially, and the periodogram analyses would then include "floating means" and "first derivatives".

Only in the short-period regime can $\gamma$ potentially get decoupled from the other parameters. For this to happen, we need dense sampling over a number of cycles. In the long-period regime, as noted below, the cross-talk never disappears and one must solve for $\gamma$, regardless of the density of sampling. The covariance of $\gamma$ with orbital parameters is easily seen here since in this regime, we only measure a portion of the orbit and in this limit, the orbit can be approximated by a linear term (constant velocity) and curvature (acceleration). The first term is covariant with the systemic motion. In the astrometry context, Black \& Scargle (1982) were the first to recognize the consequences of this covariance.

The parameters of our data set are as follows: the duration of the survey is $T_{0}, v^{\prime}\left(t_{j}\right)$ is the measured RV at epoch $t_{j}$, and $n_{0}$ is the number of measured epochs. With no loss of generality, we let our time go from $t=-T_{0} / 2$ to $t=T_{0} / 2$. This device allows us to simplify the normal 
equations (see below). To find the three unknowns, $v_{c}, v_{s}$ and $\gamma$, we minimize

$$
X^{2}=\sum_{i=0}^{n_{0}-1}\left[v^{\prime}\left(t_{i}\right)-v_{c} \cos \left(\omega t_{i}\right)-v_{s} \sin \left(\omega t_{i}\right)-\gamma\right]^{2}
$$

with respect to $v_{c}, v_{s}$, and $\gamma$. This yields a matrix equation for the three unknowns:

$$
\begin{aligned}
\left(\begin{array}{ccc}
\sum_{i=0}^{n_{0}-1} \cos ^{2}\left(\omega t_{i}\right) & \sum_{i=0}^{n_{0}-1} \cos \left(\omega t_{i}\right) \sin \left(\omega t_{i}\right) & \sum_{i=0}^{n_{0}-1} \cos \left(\omega t_{i}\right) \\
\sum_{i=0}^{n_{0}-1} \cos \left(\omega t_{i}\right) \sin \left(\omega t_{i}\right) & \sum_{i=0}^{n_{0}-1} \sin ^{2}\left(\omega t_{i}\right) & \sum_{i=0}^{n_{0}-1} \sin \left(\omega t_{i}\right) \\
\sum_{i=0}^{n_{0}-1} \cos \left(\omega t_{i}\right) & \sum_{i=0}^{n_{0}-1} \sin \left(\omega t_{i}\right) & n_{0}
\end{array}\right) \times\left(\begin{array}{c}
v_{c} \\
v_{s} \\
\gamma
\end{array}\right) \\
=\left(\begin{array}{c}
\sum_{i=0}^{n_{0}-1} v^{\prime}\left(t_{i}\right) \cos \left(\omega t_{i}\right) \\
\sum_{i=0}^{n_{0}-1} v^{\prime}\left(t_{i}\right) \sin \left(\omega t_{i}\right) \\
\sum_{i=0}^{n_{0}-1} v^{\prime}\left(t_{i}\right)
\end{array}\right) .
\end{aligned}
$$

In the short-period regime where $\tau \ll T_{0}$, we observe many cycles and thus, under most reasonable sampling schemes, the sinusoidal summations, $\sin (\omega t), \cos (\omega t)$, and $\sin (\omega t) \times \cos (\omega t)$ will average to zero, while $\sin ^{2}(\omega t)$ and $\cos ^{2}(\omega t)$ will average to $1 / 2$. Thus, in the short-period regime the matrix in Equation 6 becomes diagonal, and the fit parameters are given by

$$
\begin{gathered}
v_{c}=\frac{2}{n_{0}} \sum_{i=0}^{n_{0}-1} v^{\prime}\left(t_{i}\right) \cos \left(\omega t_{i}\right), \\
v_{s}=\frac{2}{n_{0}} \sum_{i=0}^{n_{0}-1} v^{\prime}\left(t_{i}\right) \sin \left(\omega t_{i}\right), \\
\gamma=\frac{1}{n_{0}} \sum_{i=0}^{n_{0}-1} v^{\prime}\left(t_{i}\right) .
\end{gathered}
$$

In the long-period regime, $\tau>T_{0}$, the matrix is not diagonal since most of the terms do not average to zero. However, by design (and assuming a reasonable sampling scheme), the sinusoidal summations involving only one power of $\sin (\omega t)$ will average to zero yielding the following normal equations:

$$
v_{c} \sum_{i=0}^{n_{0}-1} \cos ^{2}\left(\omega t_{i}\right)+\gamma \sum_{i=0}^{n_{0}-1} \cos \left(\omega t_{i}\right)=\sum_{i=0}^{n_{0}-1} v^{\prime}\left(t_{i}\right) \cos \left(\omega t_{i}\right)
$$




$$
\begin{aligned}
& v_{s} \sum_{i=0}^{n_{0}-1} \sin ^{2}\left(\omega t_{i}\right)=\sum_{i=0}^{n_{0}-1} v^{\prime}\left(t_{i}\right) \sin \left(\omega t_{i}\right) \\
& v_{c} \sum_{i=0}^{n_{0}-1} \cos \left(\omega t_{i}\right)+\gamma n_{0}=\sum_{i=0}^{n_{0}-1} v^{\prime}\left(t_{i}\right) .
\end{aligned}
$$

\section{Type I Errors}

Type I errors describe the probability that a high amplitude will be obtained even when no signal is present in the data. We assess the probability of type I errors, assuming that our data set consists of Gaussian noise with a mean of zero and a standard deviation of $\sigma_{0}$.

Short-Period Regime. From Equations 19 we note that for most reasonable sampling schemes, $v_{c}, v_{s}$ and $\gamma$ are simply sums of Gaussian variables and thus from the Gaussian addition theorem, all three derived parameters are also Gaussian variables. Specifically, $v_{c}$ and $v_{s}$ obey a Gaussian distribution with a mean of zero and a standard deviation of

$$
\sigma=\sqrt{\frac{2}{n_{0}}} \sigma_{0},
$$

where $n_{0}$ is the number of measurements. Denoting by $V_{1 s}$ the value of $\left|v_{c}\right|$ (or $\left.\left|v_{s}\right|\right)$ that is exceeded in $1 \%$ of cases, we note that

$$
V_{1 s}=2.61 \sigma=\frac{3.69}{\sqrt{n_{0}}} \sigma_{0} .
$$

From Equation 9 we see that $\gamma$ follows a Gaussian distribution with zero mean and a variance of $\sigma^{2} / n_{0}$. Denoting $\Gamma=|\gamma|$ we note that the $99^{\text {th }}$ percentile value of $\Gamma$ is $\Gamma_{1 s}=2.61 \sigma_{0} / \sqrt{n_{0}}$. Thus $\Gamma_{1 s}=0.71 V_{1 s}$.

Because we are interested in the fitted amplitude, we will now examine the combined statistics of $v_{c}$ and $v_{s}$. The probability density function for $v_{c}$ and $v_{s}$ is

$$
\rho\left(v_{c}, v_{s}\right) d v_{c} d v_{s}=\frac{1}{2 \pi \sigma^{2}} e^{-v_{c}^{2} / 2 \sigma^{2}} e^{-v_{s}^{2} / 2 \sigma^{2}} d v_{c} d v_{s} .
$$

Denoting by $K$ the square of the inferred amplitude, $K=v_{c}^{2}+v_{s}^{2}$, we note that the probability density function of $K$ is an exponential and find the probability to be

$$
P\left(K<K_{0}\right)=1-e^{-K_{0} / 2 \sigma^{2}} .
$$


Thus, the squared velocity amplitude that is exceeded by pure fluctuations in only $1 \%$ of the cases, is

$$
K_{1 s}=2 \sigma^{2} \ln \left(\frac{1}{1-0.99}\right)=\frac{18.42 \sigma_{0}^{2}}{n_{0}}
$$

Long-Period Regime. In this regime, the expressions for $v_{c}, v_{s}$ and $\gamma$ are not as simple as those in the short-period regime, and so we resort to simulations. To this end, we create a synthetic data set consisting of Gaussian noise with $\sigma_{0}=3 \mathrm{~m} \mathrm{~s}^{-1}$, which is the best accuracy thus far obtained for radial velocity measurements of this type (Butler et al. 1996). We sample the synthetic data at one month intervals for $T_{0}=12$ years. We explore fitted periods from 5 years to 100 years, choosing the interval between sampled periods so as to result in a 1 radian decrease in the total number of orbital cycles over the the length of the observations,

$$
\Delta \tau=\frac{\tau^{2}}{2 \pi T_{0}}
$$

Thus the sequence of the periods which we consider is $60,64,69,74,80,87,95,105,117,132,152$, 177, 212, 261, 337, 463, 699, and 1239 months.

For each period, $\tau$, we simulate $N=1000$ data sets and carry out the Least Squares fit. We set $K_{1}$ equal to the $10^{\text {th }}$ highest $K$ that arises. Clearly, $99 \%$ of the fitted $K$ 's will lie below this value. A plot of $K_{1}$ versus $\tau$ is shown in Figure 1. As Figure 1 indicates, our simulations are in excellent agreement with the expected value of $K_{1 s}$ in the short period regime (Equation 17).

Thus far, our analysis has followed that of Nelson \& Angel (1998), but at this point the two analyses diverge. Nelson \& Angel (1998) assume that $K_{1}$ in the long-period regime is a power law and from their simulations find $K_{1} \propto \tau^{3.64}$. The value of this exponent has no natural explanation and indeed it was this unusual value that motivated the present analysis.

The principal goal of our analysis was to understand the behavior of $K_{1}$ in the long-period regime and to derive an analytical expression for $K_{1}$ that is valid for all fitted periods. We begin by examining the covariance matrix of $v_{c}, v_{s}$, and $\gamma$. At short periods, we find these three fitted parameters are uncorrelated; this is expected from Equations 07 . 
However, the situation is quite different in the long-period regime as can be seen from the plots in Figure 2. From this figure we conclude the following:

1. $v_{c}$ and $v_{s}$ are correlated.

2. $v_{c}$ and $\gamma$ are anti-correlated.

3. $v_{s}$ and $\gamma$ are uncorrelated.

The corollary to this result is that phase becomes important in the long-period regime. Indeed, as can be seen from Figure 2, $\phi \sim \pm 90^{\circ}$ is preferred. Nelson \& Angel (1998) and Cumming et al. (1999) recognized that the phase would become non-random in the long period regime. However, neither they nor others have explored the full implications of this fact. Below we look into this issue in more detail.

We now provide a simple (physical) explanation of the results displayed in Figure 2. When $\tau \ll T_{0}$, sinusoids with random phase can be fit to the Gaussian data. Clearly, the amplitude of these sinusoids cannot be significantly bigger than the vertical scale of the data (which is approximately $\left.\sigma_{0}=3 \mathrm{~m} \mathrm{~s}^{-1}\right)$. However, when $\tau>T_{0}$, this is no longer necessarily the case. The maximum value of $K$ is obtained when we fit a cosine to random data since a cosine is flat around $t=0$; we remind the reader of the choice of our time baseline, $\left[-T_{0} / 2, T_{0} / 2\right]$. Recall that for small $t, \cos t \simeq 1-t^{2} / 2$. Thus, the size of the fitted cosine is limited by how much it deviates from a constant in the range from $t=\left[0, T_{0} / 2\right]$. This deviation is given by

$$
1-\cos \left(\frac{\pi T_{0}}{\tau}\right)
$$

The amplitude of this cosine is then chosen so that this deviation from a constant will be roughly equal to the vertical scale of the noise $\left(\sigma_{0}\right)$. So, Equation 19 tells us the fraction of the total fitted amplitude, and thus the actual fitted amplitude is

$$
V_{c_{1}}=\frac{2 V_{1 s}}{\left(1-\cos \left[\frac{\pi T_{0}}{\tau}\right]\right)} ; \quad \text { for } \tau \geq T_{0}
$$

here, $V_{c_{1}}$ is the value of $\left|v_{c}\right|$ which will be exceeded in $1 \%$ of Least Squares fits to Gaussian noise, $V_{1 s}$ is the corresponding $V_{c_{1}}$ in the short-period regime (Equation 14), and 2 is a normalization factor (since the above deviation was peak-to-peak). 
Now, we consider the behavior of $v_{s}$ in the long-period regime. Here, there is a slight difference: because the full amplitude of a sine centered around $t=0$ can be observed in the interval $[-\tau / 4, \tau / 4]$, the "long-period regime for $v_{s}$ " does not actually begin until $\tau>2 T_{0}$. However, once we are in the long-period regime, our analysis is much the same. In particular, we find the amplitude of a sine wave such that in the interval from $t=\left[-T_{0} / 2, T_{0} / 2\right]$, the sine wave does not exceed the vertical scale of the noise. Following the same lines as the analysis for $v_{c}$, it is clear that this amplitude must be

$$
V_{s_{1}}=\frac{V_{1 s}}{\sin \left(\frac{\pi T_{0}}{\tau}\right)} ; \quad \text { for } \tau>2 T_{0}
$$

This function is plotted with the simulated data in Figure 1 . Not surprisingly, $V_{s_{1}}$ is considerably smaller than $V_{c_{1}}$.

Understanding the behavior of $\gamma$ is not difficult in light of our understanding of $v_{c}$. Since the mean value of the simulated data is zero, our fitted function must also, in the mean, be zero. The anti-correlation between $\gamma$ and $v_{c}$ in Figure 2 confirms this expectation. From the above discussion, we know that the fitted signal is primarily a cosine with an amplitude given by Equation 20. We must choose a $\gamma$ that translates the fitted cosine such that the result is "centered" around $y=0$. This translation is accomplished by subtracting the fitted amplitude $A$ (which produces a cosine wave whose maximum value is zero) and then adding back $\sigma_{0} / 2$ in order to properly center the fitted signal. Thus, we expect

$$
\Gamma_{1}=\Gamma_{1 s}\left\{\frac{3}{1-\cos \left[\frac{\pi T_{0}}{\tau}\right]}-\frac{1}{2}\right\} .
$$

Note that we expect no correlation between $v_{s}$ and $\gamma$, because $v_{s}$ is already centered around zero, and requires no translation (or physically, a sinusoidal orbit with $\phi=0^{\circ}$ is non-degenerate with constant velocity motion).

Equipped with the behaviors of $V_{c_{1}}$ and $V_{s_{1}}$, we are now in a position to write down an 
analytical expression for $K_{1}\left(\propto V_{c_{1}}^{2}+V_{s_{1}}^{2}\right)$ at all periods:

$$
K_{1}= \begin{cases}K_{1 s}=\frac{18 \cdot 42 \sigma_{0}^{2}}{n_{0}} & \text { for } \tau \leq T_{0} \\ \frac{4 K_{1 s}}{\left(1-\cos \left[\frac{\pi T_{0}}{\tau}\right]\right)^{2}} & \text { for } T_{0}<\tau<2 T_{0} \\ \frac{5 K_{1 s}}{4}\left\{\frac{4}{\left(1-\cos \left[\frac{\pi T_{0}}{\tau}\right]\right)^{2}}+\frac{1}{\sin ^{2}\left(\frac{\pi T_{0}}{\tau}\right)}\right\} & \text { for } \tau>2 T_{0}\end{cases}
$$

Here, the factor of $5 / 4$ is chosen to enforce continuity of $K_{1}$ for all periods. Since $V_{c} \gg V_{s}$ for $\tau>T_{0}$, to a very good approximation, we can express Equation 23 as

$$
K_{1}=\frac{4 K_{1 s}}{\left(1-\cos \left[\frac{\pi T_{0}}{\tau}\right]\right)^{2}} ; \quad \text { for } \tau>T_{0}
$$

We prefer this compact formula over the more exact formula (Equation 23). As demonstrated by Figures 1 and 3 5, the analytical expressions derived above provide excellent fits to the simulated data. Thus, we have fulfilled our original objective of determining an analytical understanding of $K_{1}$.

We now turn to the importance of the phase term. As can be seen from Figure 2, in the short period regime, the distribution of $v_{c}$ and $v_{s}$ is cylindrically symmetric. However, in the long-period regime the distribution becomes highly elliptical, and the Least Squares fit overwhelmingly prefers orbits with $\phi= \pm 90^{\circ}$. An orbit sampled at a phase of $\pm 90^{\circ}$ has the smallest radial acceleration and thus for a given set of measurements yields the largest value of $K$.

We have so far considered only the statistics of $K$, which is perfectly reasonable when the $v_{c}-v_{s}$ distribution is cylindrically symmetric. However, in the long-period regime this distribution is elliptical, in which case the exclusive use of the radial parameter $K$ is bound not to be optimal. Thus in the long-period regime, we need to look at both the $v_{c}$ and $v_{s}$, or equivalently the phase and amplitude of the fitted parameters.

To this end, we define ellipses in the $v_{c}-v_{s}$ plane, $\epsilon_{1}$, such that $1 \%$ of simulated fitted pairs lie outside this ellipse. The parameters of these ellipses are easily obtained since we have analytical expressions for $V_{c_{1}}$ and $V_{s_{1}}$ (Equations 20 and 21). Further discussion and exploitation of this "amplitude-phase" $(K-\phi)$ analysis is postponed to Section 4 . 
Uneven and Sparse Sampling. We re-iterate that the above results have been derived under the assumption of dense, even sampling. Here, we examine the validity of these results in the case of random sampling, paying particular attention to the regime where $n_{0}$ is small.

As discussed in Section 2.1, Equations $14-17$ are only valid if the chosen sampling scheme preserves the independence of $v_{c}, v_{s}$, and $\gamma$. If we have a large number of samples over a number of cycles, then even if our sampling is completely random, we expect that the dense sampling of the sinusoidal summations will preserve the diagonality of Equation 6 (and hence Equations 79 will hold). However, for sparse sampling (i.e. small $n_{0}$ ), our choice of sampling scheme becomes important. In particular, if we sample the data evenly in the interval $\left[-T_{0} / 2, T_{0} / 2\right]$, then Equation 6 remains diagonal, and the independence of $v_{c}, v_{s}$, and $\gamma$ is preserved. In contrast, if the data are sparsely and unevenly sampled, then the summations of $\sin (\omega t), \cos (\omega t)$, and $\sin (\omega t) \times \cos (\omega t)$ may no longer average to zero, and $v_{c}, v_{s}$, and $\gamma$ will be covariant. Physically, this corresponds to the fact that for a small number of randomly spaced samples, the sampled sinusoid might look like a straight line (e.g. if all the samples happen to lie at the zero-crossings of the sine-wave). Thus, for sparse and uneven sampling, Equations $14-17$ do not accurately describe the sensitivity of the Least Squares fit.

We verify these assertions through simulations: for $n_{0}=\{5, \ldots, 25\}$, we simulate $N=1000$ data sets and do Least Squares fits to sinusoids with $\tau=0.2 T_{0}$. The sampling times are drawn from a distribution given by $t_{j} \epsilon[\Delta t \times(j-R), \Delta t \times(j+R)]$. Here, $\Delta t$ denotes the sampling interval, given by $T_{0} / n_{0}$, and $R$ is a parameter describing the unevenness of the sampling. $R=0$ gives an even sampling scheme, and $R=1 / 2$ gives random sampling. The results of these simulations are shown in Figure 6 . We see that for $n_{0} \lesssim 10$, one suffers substantial losses in sensitivity if the data are unevenly sampled. This phenomenon was noted by Cumming et al. (1999) . However, the basic reason why we see this phenomenon is because Equation 17 is no longer valid. This discussion re-emphasizes of the generality and robustness of the Least Squares approach over the periodogram approach.

We now repeat the above simulations using fitted sinusoids with $\tau=10 T_{0}$, to explore the 
effects of sparse and uneven sampling in the long-period regime. The results of this simulation are shown in Figure 7. From this figure we see that in the long-period regime, the evenness of the sampling scheme is not nearly as important as it is in the short-period regime. This is to be expected, because our analysis of the behavior of $K_{1}$ in the long-period regime takes into account the covariance between $v_{c}, v_{s}$, and $\gamma$, and thus we expect it to be applicable to sparsely and unevenly sampled data.

We end this section by acknowledging that our treatment of the statistics is not accurate in the low $n_{0}$ regime. At the very least one needs to be aware of the loss of the degrees of freedom because three parameters are obtained from the data. The correct value of the degrees of freedom will depend on what statistic is being estimated. This issue is not central to the main goal of this paper and we intend to investigate this issue in a later paper.

\section{Type II Errors}

So far we have computed the probability of detecting an apparent signal generated purely by noise. In the language of inference, we have discussed Type I probabilities. Our analysis was centered on obtaining the statistics of $K$, the squared amplitude from the fitted parameters $v_{c}$ and $v_{s}$. In particular, we estimated $K_{1}$, the $99^{\text {th }}$ percentile of $K$ (see Equation 24). However, following this analysis, we noted that in the long-period regime, $v_{c}$ and $v_{s}$ form an elliptical distribution, and thus merely looking at the statistics of $K$, a radial parameter, was not optimal.

We now consider Type II probabilities - the probability of failing to detect a genuine signal due to contamination by noise. The goal of this section is to understand the statistics of $K$ in the presence of both signal and noise. To this end, we simulate a data set that consists of signal and noise:

$$
v^{\prime}\left(t_{i}\right)=\sqrt{K} \sin \left(\frac{2 \pi t_{i}}{\tau}+\phi\right)+N\left(t_{i}\right)
$$

where $\sqrt{K}$ is the amplitude of the signal, and $N\left(t_{i}\right)$ is the Gaussian noise. We let $\phi$ be drawn from a uniform distribution in the interval from $[0,2 \pi]$, an appropriate assumption for circular 
orbits. We choose an initial signal amplitude of $\sigma_{0} / 2$, and then do $N=1000$ Least Squares fits (with the same parameters as in Section 3).

\subsection{Amplitude-Only Analysis}

Following the path used for Type I errors, we will first base our analysis on $K$, i.e. we will ignore the issue of the elliptical nature of the $v_{c}-v_{s}$ distribution. At each period, we determine how many of the fitted amplitudes lie below $K_{1}$. If it is less than $0.01 N$, then we have found the value of $K$ such that $99 \%$ of the fitted amplitudes lie above $K_{1}$. We call this $K_{99}$. If, however,

more than $1 \%$ of the fitted amplitudes lie below $K_{1}$, we increment the signal amplitude by $K^{0.6} / 20$ until we find $K_{99}$. A plot of $K_{99} / K_{1}$ versus period is shown in Figure 8 .

We note that our choice of $99 \%$ confidence for $K$ is arbitrary and also very conservative. Most observers would be keen to make a discovery rather than set stringent upper limits. Thus one may wish to consider $K_{50}$ (or $K_{90}$ ), which gives the signal amplitude necessary to be detected $50 \%$ (or $90 \%$ ) of the time. Plots of $K_{90}$ and $K_{50}$ versus period are shown in Figure 9 and Figure 10, respectively.

\subsection{Amplitude-Phase Analysis}

In Section 3, we showed that in the long-period regime, the $v_{c}-v_{s}$ distribution is elliptical. In view of this, the phase of the signal in Equation 25 is critical. This point is best understood in the results of the simulations displayed in Figure 11 for five cases: (a) no signal, (b)-(d), a signal with amplitude $\sqrt{K_{1}}$ (at that period) and $\phi=0^{\circ}, 45^{\circ}, 90^{\circ}$, respectively, and (e) a signal with amplitude of $\sqrt{K_{1}}$ and the phase being randomly (for each simulation) chosen from the range $[0,2 \pi]$ (uniform probability density function).

In Figure 11, the amplitude-only analysis would consider all points that lie inside the circle of radius $K_{1}$ to be indistinguishable from those produced by noise. However, in the framework 
of the $K-\phi$ analysis, we would consider all points within the ellipse to be indistinguishable from those produced from noise. The superiority of the $K-\phi$ analysis is evident given that the size of the ellipse is smaller than that of the circle. This superiority is reflected numerically as follows: the fraction of simulations in which the signal is reliably recovered by the $K-\phi$ analysis is (100\%, $100 \%, 33 \%, 95 \%$ ) for cases (b-e) whereas for the amplitude-only analysis the corresponding fractions are $(25 \%, 35 \%, 33 \%, 34 \%)$.

A comparison of Figures 810 shows the increasing gain of the $K-\phi$ analysis as the tolerance for committing type II error is increased. When the Type II probability is set to $99 \%$, the $K-\phi$ analysis lowers the $K$ threshold by $20 \%$ whereas if we are willing to accept a $50 \%$ type II error probability then the $K$ threshold is lowered by nearly a factor of 30 !

\subsection{Linear Analysis of Long Term Trends}

Above we illustrated the superiority of an amplitude-phase analysis over an amplitude-only analysis. However, some previous authors (Walker et al. 1995; Cumming et al. 1999) have analyzed long-term trends in the data (i.e. apparent signals with $\tau \gg T_{0}$ ) by examining the slope of the best-fit straight line, denoted by $a$. This best-fit line is subtracted from the data, and an amplitude-only analysis is subsequently performed on the residuals. Here, we discuss this "slope analysis" in the context of the results of Sections 4.1 and 4.2 .

To determine the sensitivity of slope analysis, we simulate 1000 data sets with Gaussian noise of zero mean and $\sigma_{0}=3 \mathrm{~m} \mathrm{~s}^{-1}$, sampled at one month intervals for $T_{0}=12$ years. For each simulated data set, we perform a Least Squares fit to a straight line, given by $a t+b$. The $99^{\text {th }}$ percentile slope, denoted by $A_{1}$, is determined by finding the value of $|a|$ such that $99 \%$ of simulated data sets yield $|a|<A_{1}$. Next, we determine the type II errors, by injecting a sinusoidal signal into the simulated data (Equation 25) and finding the necessary squared signal amplitude $(K)$ such that the signal can be reliably recovered. This analysis is the same as that carried out in Section 4.1 except that instead of using the amplitude of fitted sinusoids, we use the slope of 
fitted straight lines to set confidence limits. Thus, at each sampled period, we can distinguish a real detection from one produced by noise if the fitted $|a|$ is greater than $A_{1}$.

The results of these simulations are shown in Figures 8 10. At sampled periods where the amplitude-only analysis is more sensitive than the slope analysis, only the former is shown. We note that although the slope analysis may be more sensitive than the amplitude-only analysis (in the long-period regime), the amplitude-phase analysis still yields a significant improvement. Moreover, the discrepancy between the amplitude-phase and slope analyses is largest for $\tau \lesssim 3 T_{0}$. We examine this discrepancy further by performing an analysis analogous to that shown in Figure 11e. In particular, for fitted periods of $\tau=2 T_{0}$ and $\tau=10 T_{0}$, we inject simulated data sets consisting of sinusoids of random phase and $K=K_{1}$ plus Gaussian noise. The percentage of signals recovered by the amplitude-only, amplitude-phase, and slope analyses (respectively) is $(57 \%, 87 \%, 59 \%)$ for $\tau=2 T_{0}$, and $(34 \%, 95 \% 91 \%)$ for $\tau=10 T_{0}$.

We can understand the relative sensitivities of the $K-\phi$ and slope analyses by noting that the slope analysis is an explicitly linear technique, and thus it throws away any information that is contained in the curvature of the sine or cosine components of the fitted sinusoid. The $K-\phi$ analysis, in contrast, utilizes all of the information contained in the linear and curvature components of the fitted sinusoid. These curvature components will tend to zero for $\tau \gg T_{0}$, and thus in this regime the sensitivity of the slope analysis will approach that of amplitude-phase analysis. However, for $\tau \sim 2-3 T_{0}$, these curvature terms are significant, and the $K$ - $\phi$ analysis yields a substantial improvement over a slope analysis.

The above discussion (in our view) re-emphasizes the generality of the Least Squares approach. While Walker et el. (1995) and Cumming et al. (1999) introduce a modified periodogram to account for a slope in the data, the Least Squares approach can be applied without modification, as it has been in $\S 4.2$. 


\section{Conclusions}

We have developed an analytical understanding of the sensitivity of the radial velocity technique of planet detection in the regime where the orbital period is longer than the total baseline of the observations. We also examined the sensitivity in the short-period regime, paying particular attention to the case of sparsely sampled data. Moreover, we have illustrated the benefits of Least Squares fitting over the equivalent, but more complicated technique of periodogram analysis; while the periodogram must be modified to deal with long-period signals, or with sparsely sampled data, the Least Squares approach can be applied in its basic form.

We have also discovered the potentially exciting new result that in the long-period regime one obtains additional information from the phase. Analyses of the RV data to date (e.g. Walker et al. 1995; Nelson \& Angel 1998; Cumming et al. 1999) have been based either on amplitude-only analyses, or on amplitude analyses supplemented by slope analyses. However, as dramatically demonstrated by Figures 9 and 10, $K-\phi$ analysis has significant advantages over these previous analyses, especially in the interesting regime where $\tau \sim 2-3 T_{0}$.

Thus, we propose using a confidence test based on amplitude and phase, characterized by ellipses in the $v_{c}-v_{s}$ plane, $\epsilon_{1}$. The analytical expressions we have developed for $V_{c_{1}}$ and $V_{s_{1}}$ provide the analytical behavior of $\epsilon_{1}$ as a function of period. For real RV data, like those mentioned above, we suggest that the fitted $v_{c}$ and $v_{s}$ (at each period) be compared to the $\epsilon_{1}$ ellipses. Points lying outside these ellipses would signify the detection of periodic signals.

We have applied this $K-\phi$ analysis technique to the RV data of Walker et al. (1995). While Nelson \& Angel (1998) examined this data with an amplitude-only analysis and reported several marginal detections, our technique yielded several clear detections of periodic signals. Although we cannot say whether these periodicities represent planetary signals (as opposed to stellar cycles or periodic systematic errors), it is clear that the use of amplitude and phase allows previously unknown periodicities to be detected. In the future, we hope to apply this $K-\phi$ analysis to more comprehensive RV surveys, and to detect long-period companions. 


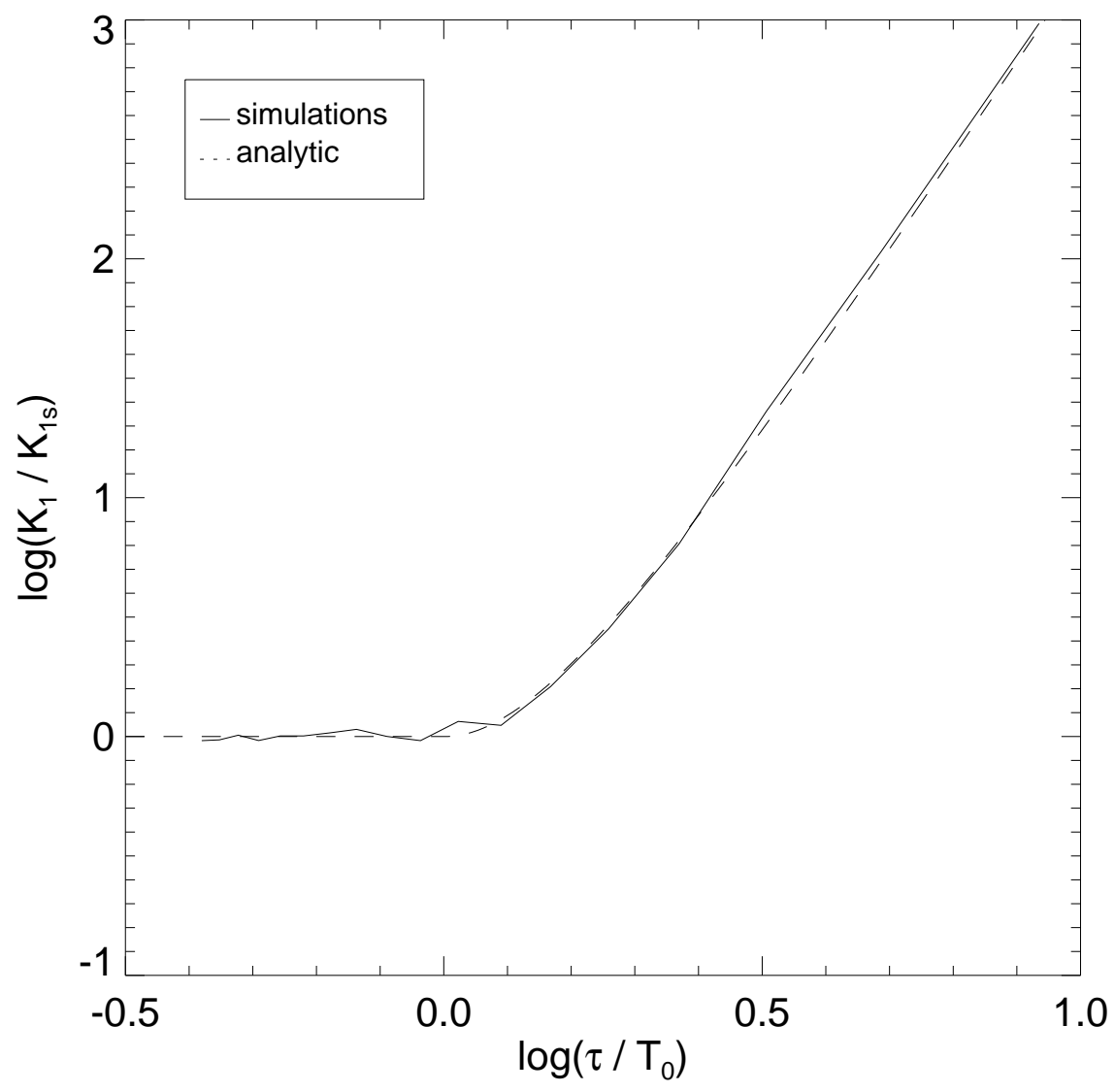

Fig. 1.- A plot of $\log \left(K_{1} / K_{1 s}\right)$ versus $\log \left(\tau / T_{0}\right)$ in the long-period regime of the radial velocity technique (solid line). $K_{1}$ is the 99 th percentile of $K=v_{c}^{2}+v_{s}^{2}$. Here, we simulated $N=1000$ data sets with Gaussian noise of zero mean and $\sigma_{0}=3 \mathrm{~m} \mathrm{~s}^{-1}$. The data were sampled at one month intervals for $T_{0}=144$ months. The dashed line shows the analytical $K_{1}$ predicted by Equation 23 . 

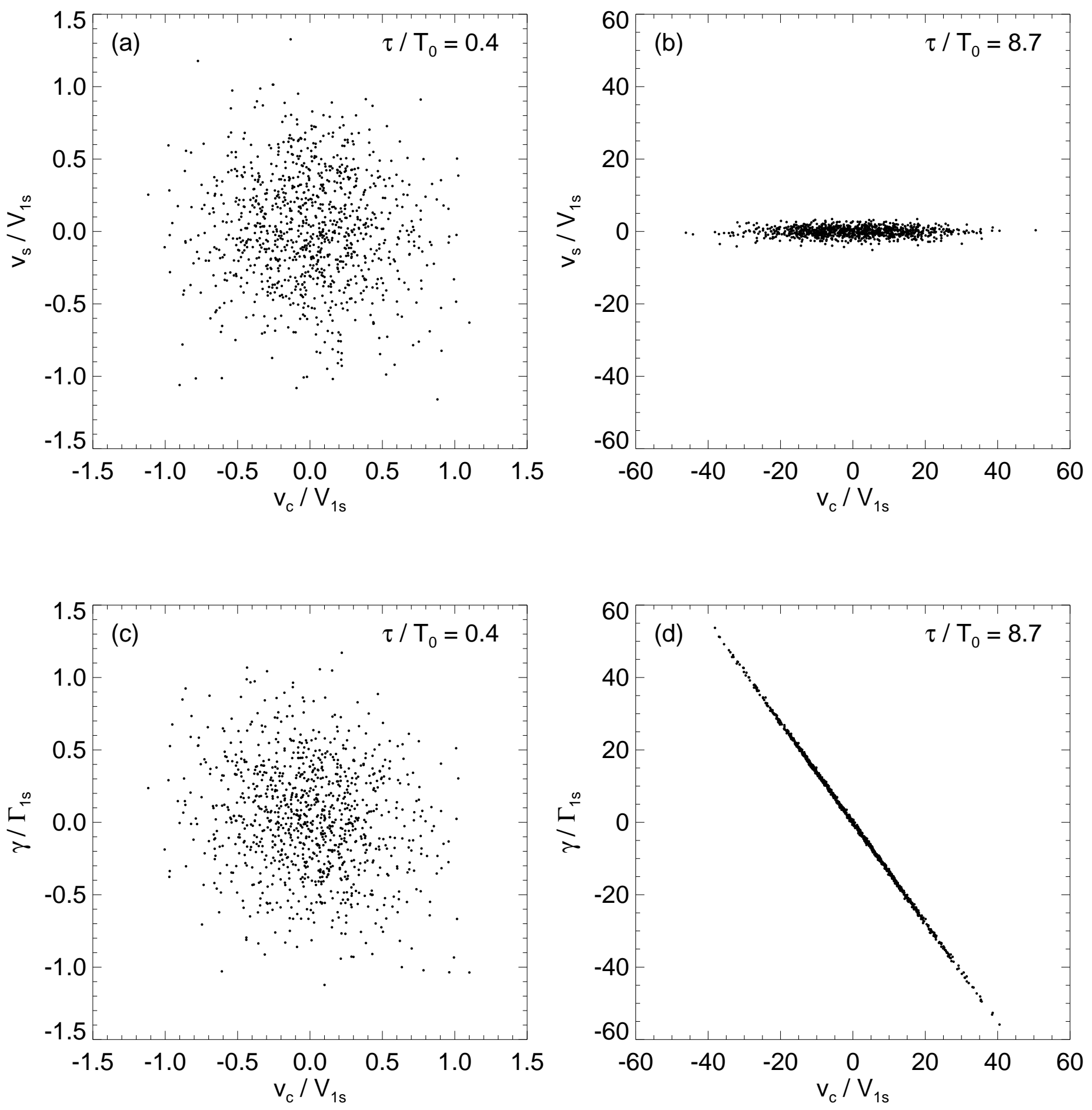

Fig. 2.- Plots of $v_{c}$ versus $v_{s}$ and $v_{c}$ versus $\gamma$ in the short and long-period regimes. In both cases, the duration of the RV monitoring is $T_{0}=12 \mathrm{yr}$. In the short-period regime, $\tau \ll T_{0}, v_{c}, v_{s}$, and $\gamma$ are uncorrelated (a),(c). In the long-period regime, strong correlations are seen. In particular, we see that orbital phases of approximately $\pm 90^{\circ}$ are preferred (b) and that $v_{c}$ and $\gamma$ are strongly anti-correlated (d). 


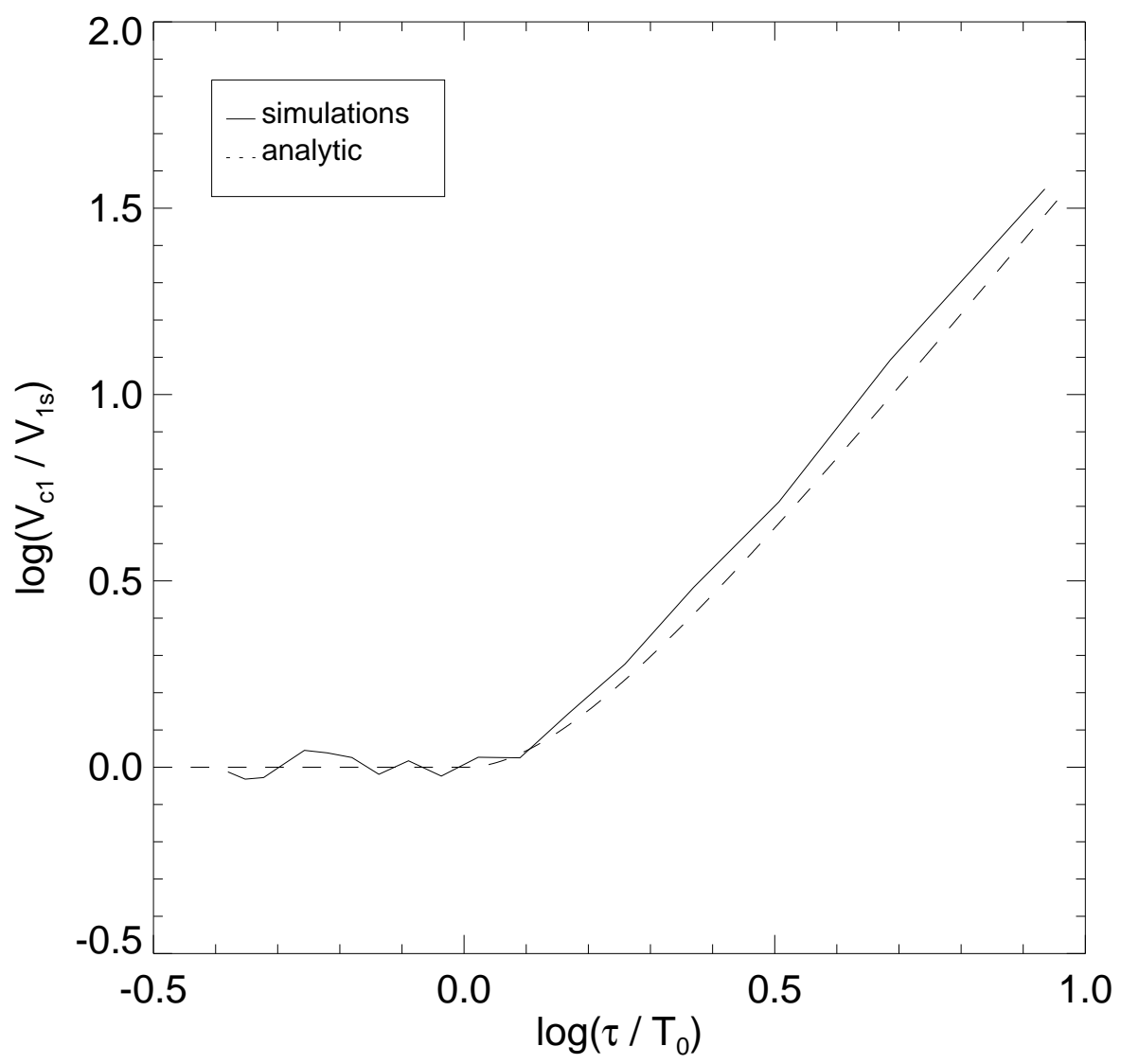

Fig. 3.- A plot of $\log \left(V_{c_{1}} / V_{1 s}\right)$ versus $\log \left(\tau / T_{0}\right) . V_{c_{1}}$ is the value of $\left|v_{c}\right|$ that is exceeded in $1 \%$ of the simulations and $V_{1 s}$ is a normalizing factor (see Equation 14). See the caption of Figure 1 for details of the simulations. The solid line shows the behavior of the simulated data, and the dashed line represents the analytical expression from Equation 20. 


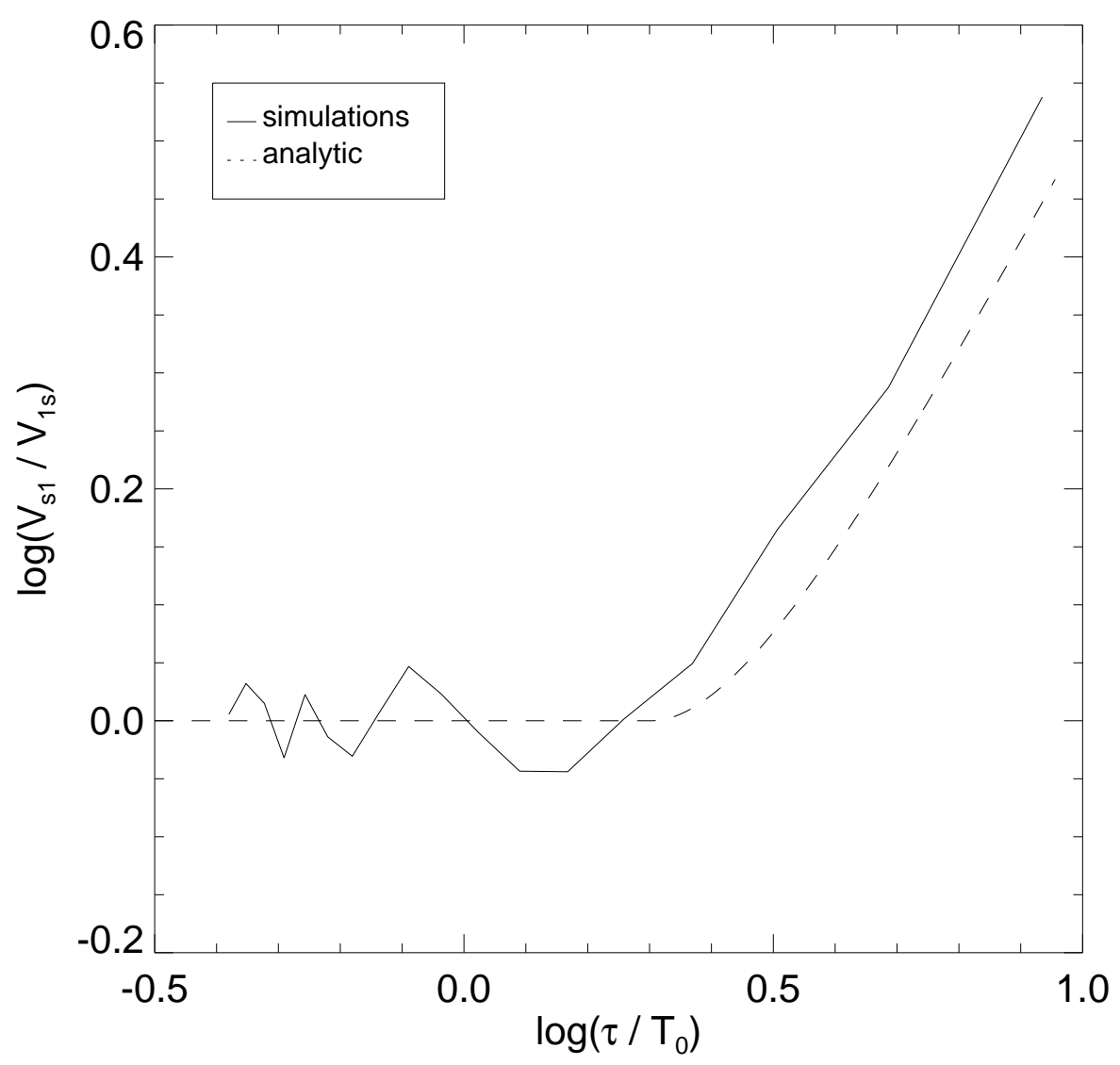

Fig. 4.- A plot of $\log \left(V_{s_{1}} / V_{1 s}\right)$ versus $\log \left(\tau / T_{0}\right) . V_{s_{1}}$ is the value of $\left|v_{s}\right|$ that is exceeded in $1 \%$ of the simulations. See caption to Figure 1 for details of the simulations. The solid line shows the behavior of the simulated data, and the dashed line represents the analytic expression from Equation 21. Bearing in mind that the vertical scale of this figure is quite fine, we note that the analytical expression is a good fit to the simulated data. 


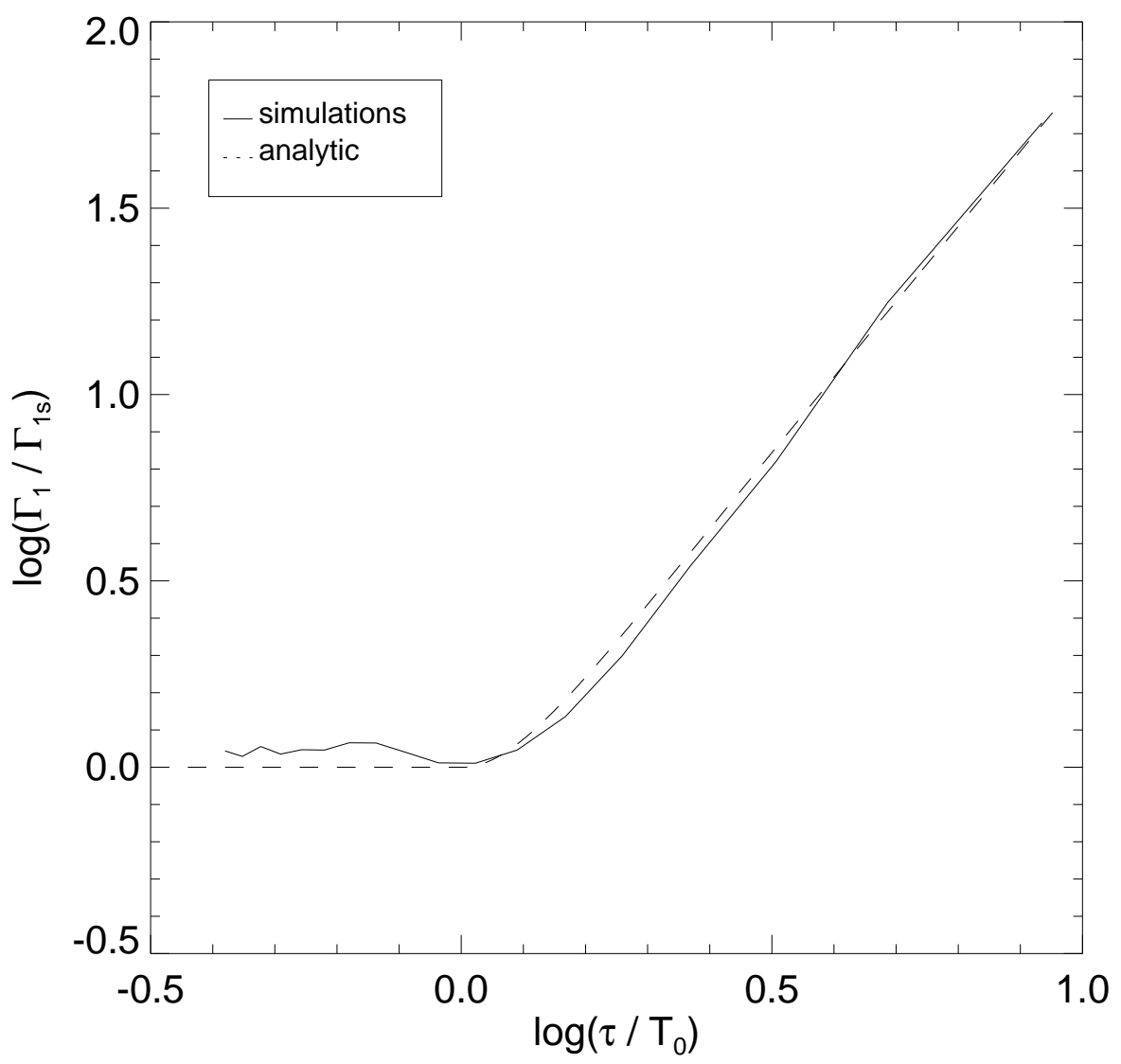

Fig. 5.- A plot of $\log \left(\Gamma_{1} / \Gamma_{1 s}\right)$ versus $\log \left(\tau / T_{0}\right) . \quad \Gamma_{1}$ is the value of $|\gamma|$ that is exceeded in $1 \%$ of the simulations. See caption to Figure 1 for details of the simulations. The solid line shows the behavior of the simulated data, and the dashed line represents the analytic expression from Equation 22. 


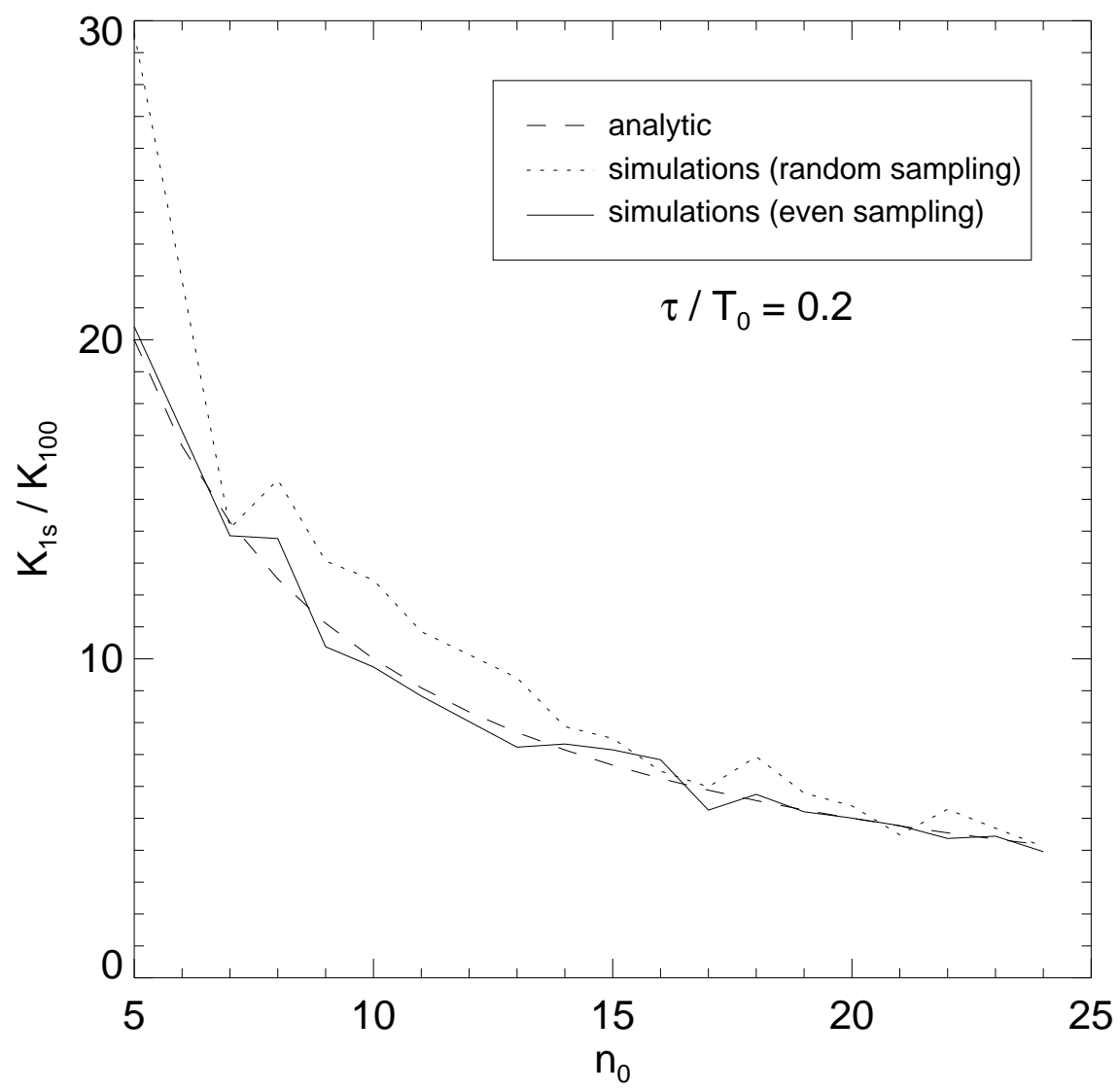

Fig. 6.- A plot of $K_{1 s}$ versus $n_{0}$ for evenly sampled (solid line) and randomly sampled simulated data (dotted line). (For details of the simulations, see the caption to Figure 1.) The analytical expression given by Equation 17 is indicated by the dashed line. All of these curves are normalized by the value of $K_{1 s}$ for $n_{0}=100$, denoted by $K_{100}$. We note that for $n_{0} \lesssim 10$, the inclusion of $\gamma$ in the model equation is important for randomly sampled data, and that regardless of the chosen sampling scheme, we suffer significant losses in sensitivity. 


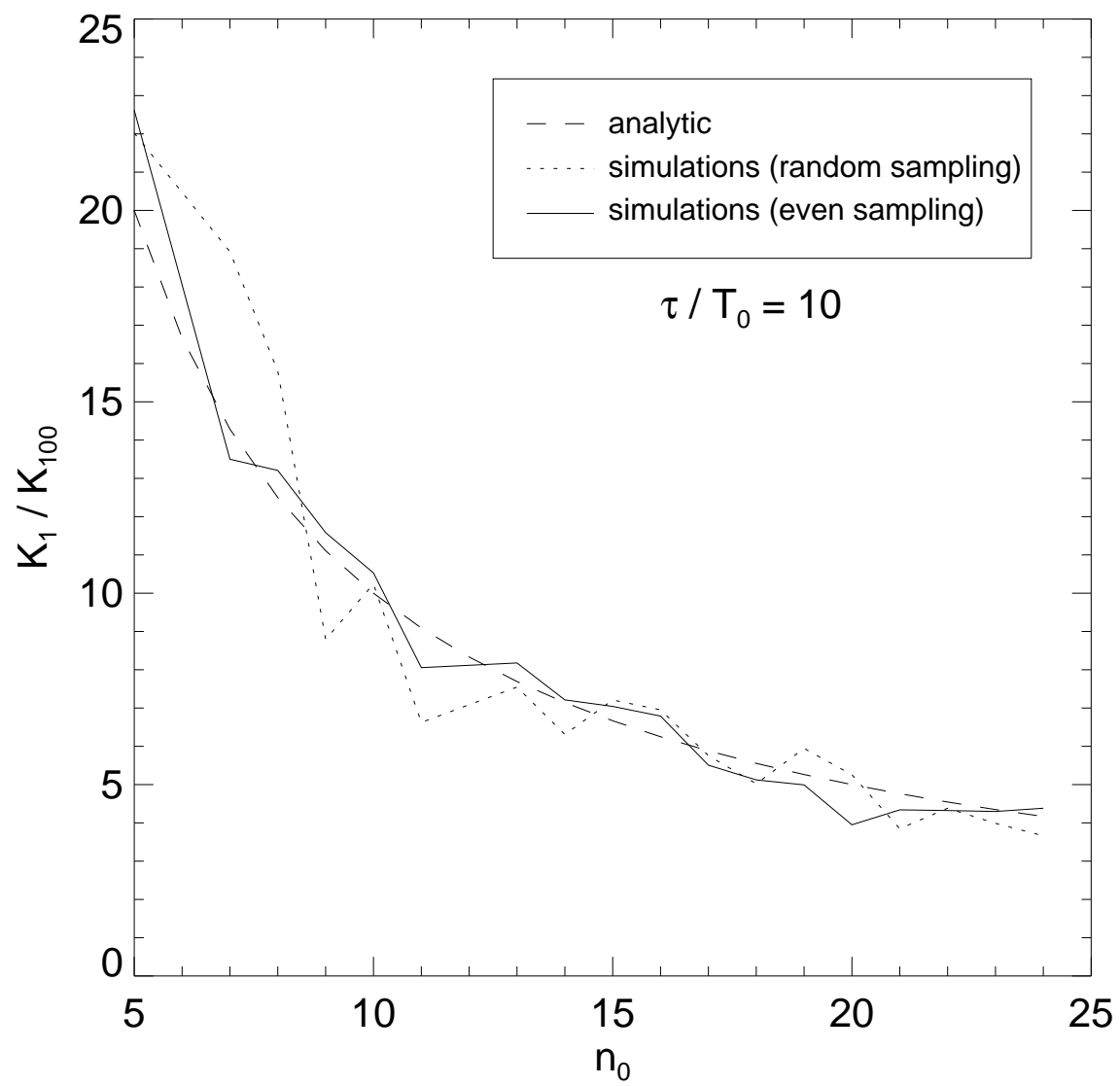

Fig. 7.- A plot of $K_{1}$ versus $n_{0}$ for evenly sampled (solid line) and randomly sampled simulated data (dotted line). (For details of the simulations, see the caption to Figure 1.) The analytical expression given by Equation 24 is indicated by the dashed line. All of these curves are normalized by the value of $K_{1}$ for $n_{0}=100$, denoted by $K_{100}$. We note that the difference between even and random sampling is not overly important, as we expect because the behavior of $K_{1}$ in the longperiod regime (Equation 24) was derived under the assumption that $v_{c}, v_{s}$, and $\gamma$ are covariant. 


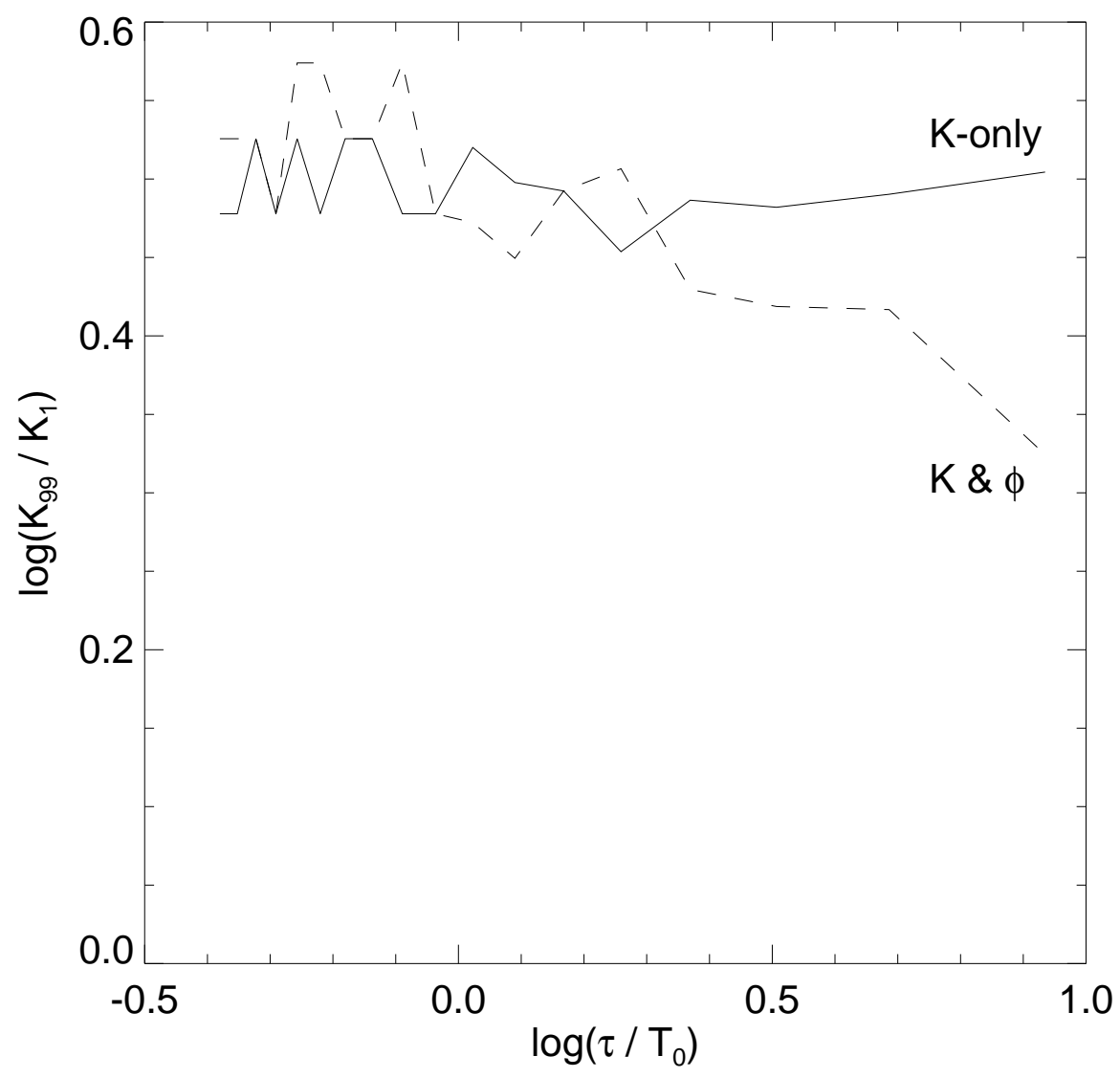

Fig. 8.- A plot of $\log \left(K_{99} / K_{1}\right)$ versus $\log \left(\tau / T_{0}\right)$ as determined in the amplitude-only and $K-\phi$ cases. For the amplitude-only case (solid line), $K_{99}$ represents the squared signal amplitude such that $99 \%$ of the simulated data yield fitted $K$ 's greater than $K_{1}$. For the $K-\phi$ analysis, $K_{99}$ is the necessary amplitude such that $99 \%$ of fitted $K$ and $\phi$, or equivalently $\left\{v_{c}, v_{s}\right\}$, lie outside of $\epsilon_{1}$ (the $\epsilon_{1}$ ellipse contains $99 \%$ of fits to noise-only data; for further details see the discussion towards the end of Section (3). The $99^{\text {th }}$ percentile of the slope analysis (discussed in Section 4.3) is greater than the amplitude-only $K_{99}$ for all sampled periods, and thus is not shown here. At each period, we carried out 10,000 simulations; the phase of the signal was assumed to be randomly and evenly distributed over the range $[0,2 \pi]$. 


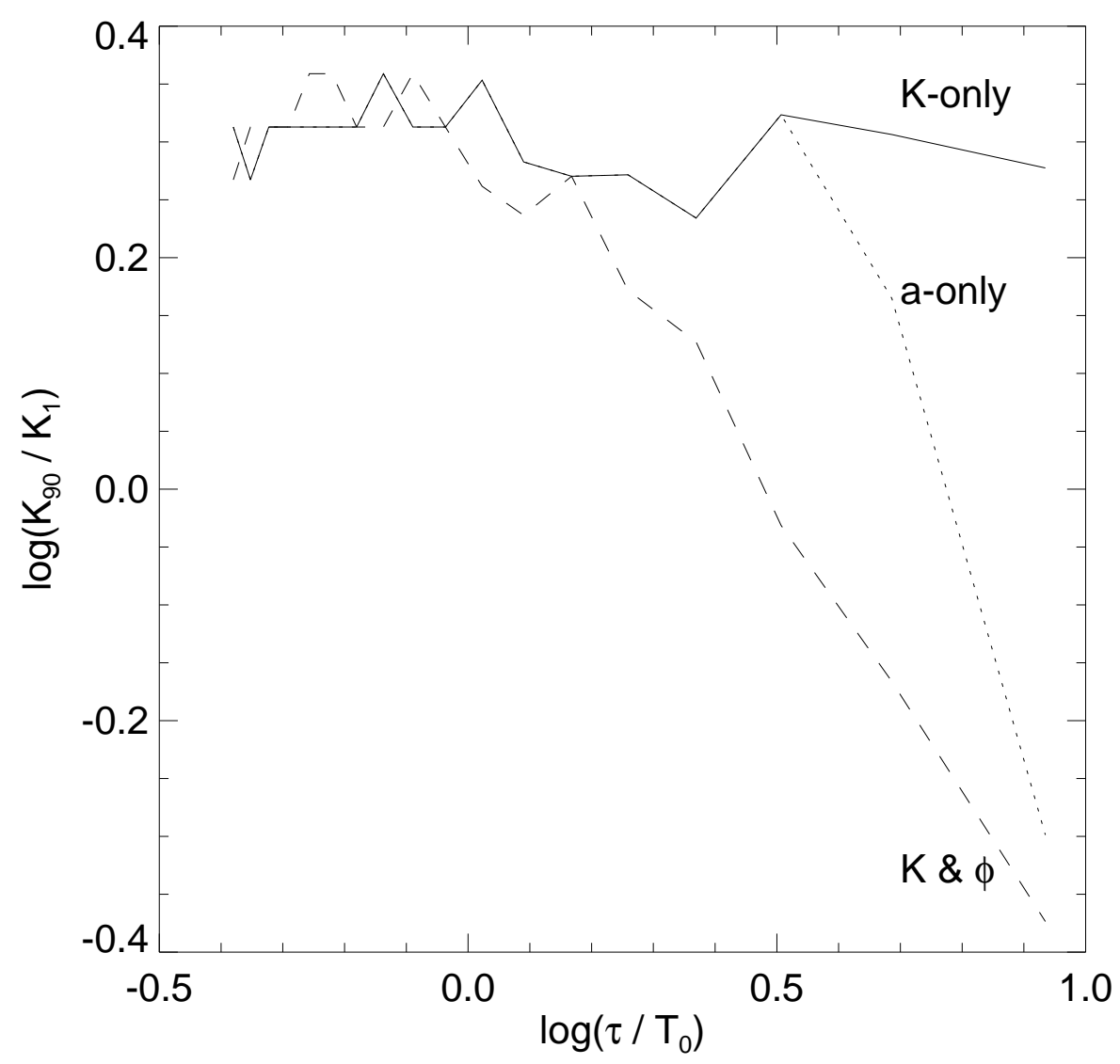

Fig. 9.- A plot of $\log \left(K_{90} / K_{1}\right)$ versus $\log \left(\tau / T_{0}\right)$ as determined in the amplitude-only (solid line), $K-\phi$ (dashed line), and slope-only (dotted line) cases. At sampled periods where the amplitudeonly $K_{90}$ is less than the slope-only $K_{90}$, only the former is shown. $K_{90}$ represents the squared signal amplitude such that $90 \%$ of the simulated data yield fitted coefficients greater than the type I sensitivity limits (at that period), given by $K_{1}, \epsilon_{1}$, and $A_{1}$ for the $K$-only, $K$ - $\phi$, and $a$-only analyses, respectively. See the caption to Figure 8 for further details. 


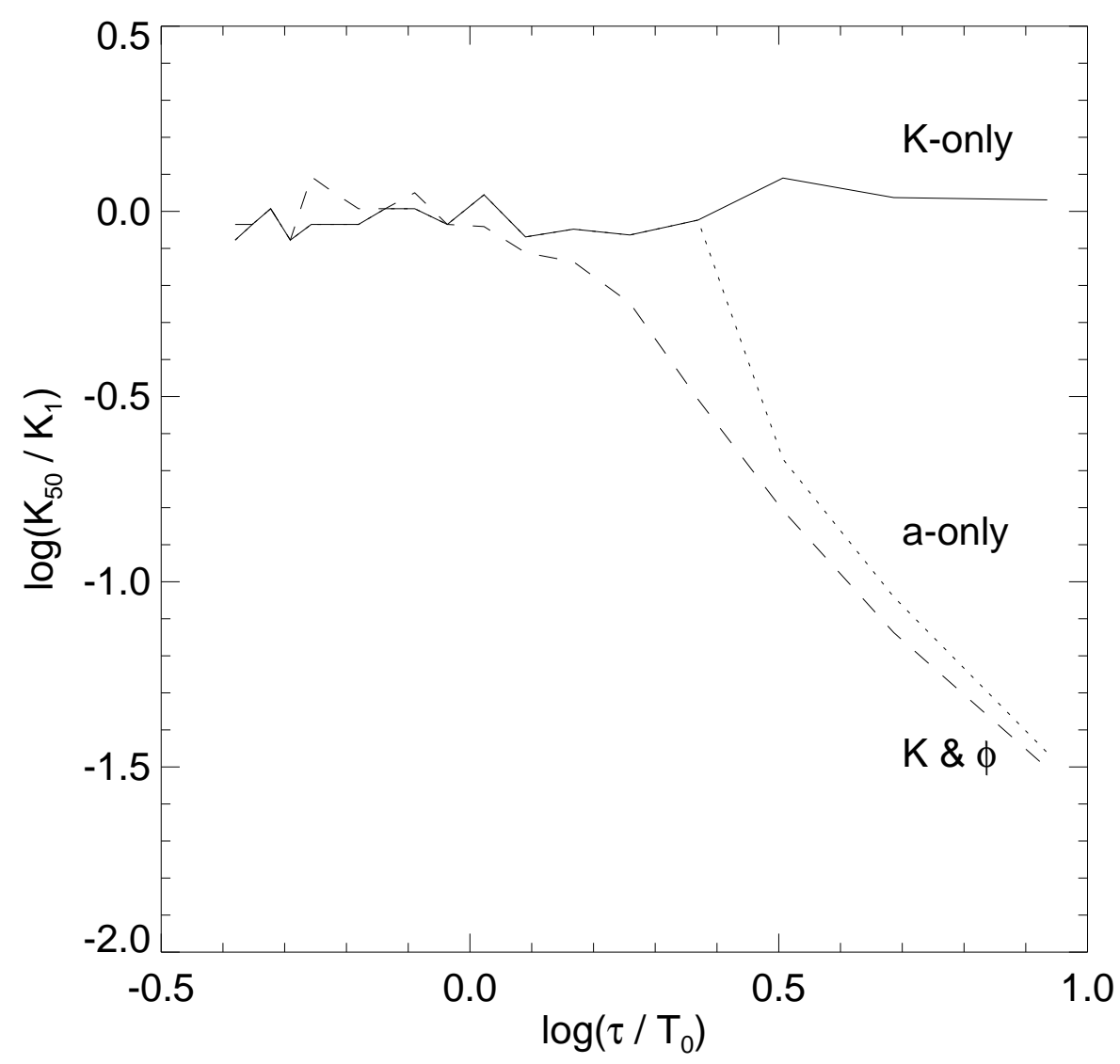

Fig. 10. - A plot of $\log \left(K_{50} / K_{1}\right)$ versus $\log \left(\tau / T_{0}\right)$ as determined in the amplitude-only case (solid line), the $K-\phi$ case (dashed line), and the slope-only case (dotted line). At sampled periods where the amplitude-only $K_{50}$ is less than the slope-only $K_{50}$, only the former is shown. $K_{50}$ represents the squared signal amplitude such that $50 \%$ of the simulated data yield fitted coefficients greater than the type I sensitivity limits (at that period), given by $K_{1}, \epsilon_{1}$, and $A_{1}$ for the $K$-only, $K$ - $\phi$, and $a$-only analyses, respectively. See the caption of Figure 8 for further details. 

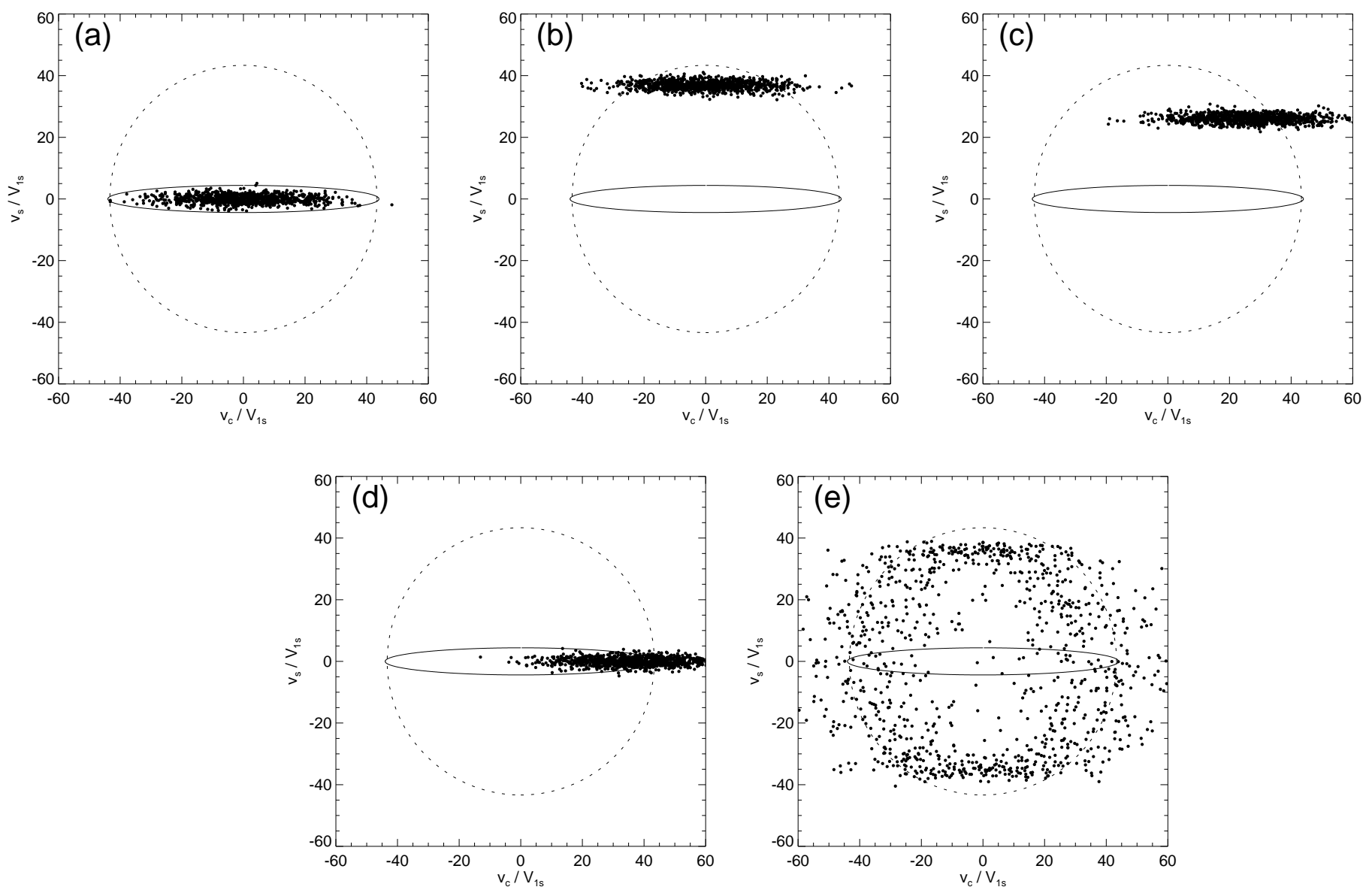

Fig. 11. - Distribution of the fitted parameters, $\left\{v_{c}, v_{s}\right\}$ for $\log \left(\tau_{0} / T_{0}\right)=1.0$. A total of $N=1000$ simulations were performed for five different assumptions. (a) No signal is present. (b)-(e) show data sets containing signals with an amplitude of $\sqrt{K_{1}}$, and $\phi=0^{\circ}, 45^{\circ}, 90^{\circ}$, and random phase, respectively. $\epsilon_{1}$ and the circle of radius $K_{1}$ are also plotted in (a)-(e). (a) shows that $99 \%$ of the fitted data points lie within $\epsilon_{1}$. (b)-(e) demonstrate the importance of phase in the determination of Type II errors: for signals with phases different than $\pm 90^{\circ}$, the fitted $\left\{v_{c}, v_{s}\right\}$ have a significant component along the $v_{s}$-axis, causing them to lie outside of $\epsilon_{1}$. Thus, the fraction of signals detected by the $K-\phi$ analysis is $(100 \%, 100 \%, 33 \%, 95 \%)$ for cases (b)-(e), while the corresponding fractions for the amplitude-only analysis are $(25 \%, 35 \%, 33 \%, 34 \%)$. 


\section{REFERENCES}

Black, D.C., \& Scargle, J.D., 1982, Ap. J., 263, 854.

Boss, A.P., 1995, Science, 267, 360.

Butler, P., Marcy, G., Williams, E., Hauser, H., \& Shirts, P., 1997, Ap. J. (Letters), 474, L115.

Butler, P., \& Marcy, G., 1996, Ap. J. (Letters), 464, L153.

Butler, P., Marcy, G., Williams, E., McCarthy, C., \& Vogt, S., 1996, PASP, 108, 500.

Cumming, A., Marcy, G.W., \& Butler, R.P., 1999, Ap. J., 526, 890.

Fischer, D., Marcy, G., Butler, P., Vogt, S., \& Apps, K., 1998, PASP, 111, 50.

Horne, J.H., \& Baliunas, S.L., 1986, Ap. J., 302, 757.

Cochran, W. D., Hatzes, A. P., Butler, R. P. \& Marcy, G. W., 1997, Ap. J., 483, 457.

Lomb, N.R., 1976, Ap. Space Sci., 39, 447

Marcy, G., Butler, P., Vogt, S., Fischer, D., \& Lissauer, J., 1998, Ap. J. (Letters), 505, L147.

Marcy, G., Butler, P., Vogt, S., Fischer, D., \& Liu, M., 1998, Ap. J., 520, 239.

Marcy, G., \& Butler, P., 1996, Ap. J. (Letters), 464, L147.

Mayor, M., Queloz, D., Beuzit, J.-L., Mariotti, J.-M., Naeff, D., Perrier, C., \& Sivan, J.-P., 1998, Protostars and Planets (V. Mannings ed.)

Mayor, M., \& Queloz, D., 1995, Nature, 378, 355.

Nelson, A.F., \& Angel, J.R.P., 1998, Ap. J., 500, 940.

Noyes, R., Jha, S., Korzennik, S., Krockenberger, M., Nisenson, P., Brown, T., Kennelly, E., \& Horner, S., 1997, Ap. J. (Letters), 483, L111. 
Queloz, D., Mayor, M., Weber, L., Naeff, D., Udry, S., Santos, N., Blecha, A., Burnet, M., \& Confino, B., 1999, A\&A, , submitted.

Scargle, J.D., 1982, Ap. J., 263, 835.

Walker, G.A.H., Walker, A.R., Irwin, A.W., Larson, A.M., Yang, S.L.S., \& Richardson, D.C., 1995, Icarus, 116, 359.

This manuscript was prepared with the AAS LATEX macros v4.0. 